Portland State University

PDXScholar

Physics Faculty Publications and Presentations

Physics

$2-1-2008$

\title{
Dark Current Measurements in a CMOS Imager
}

William C. Porter

Bradley Kopp

Justin Charles Dunlap

Portland State University

Ralf Widenhorn

Portland State University

Erik Bodegom

Portland State University

Follow this and additional works at: https://pdxscholar.library.pdx.edu/phy_fac

Part of the Physics Commons

Let us know how access to this document benefits you.

\section{Citation Details}

William C. Porter ; Bradley Kopp ; Justin C. Dunlap ; Ralf Widenhorn and Erik Bodegom, "Dark current measurements in a CMOS imager", Proc. SPIE 6816, Sensors, Cameras, and Systems for Industrial/ Scientific Applications IX, 68160C (February 29, 2008); doi:10.1117/12.769079

This Article is brought to you for free and open access. It has been accepted for inclusion in Physics Faculty Publications and Presentations by an authorized administrator of PDXScholar. Please contact us if we can make this document more accessible: pdxscholar@pdx.edu. 


\title{
Dark current measurements in a CMOS imager
}

\author{
William C. Porter, ${ }^{a}$ Bradley Kopp, ${ }^{a}$ Justin C. Dunlap, ${ }^{a}$ Ralf Widenhorn,,${ }^{a, b}$ and Erik Bodegom ${ }^{\mathrm{a}, \mathrm{b}}$ \\ ${ }^{\mathrm{a} P o r t l a n d}$ State, Portland, OR 97207 and ${ }^{\mathrm{b}}$ Digital Clarity Consultants, Lyons, OR 97328
}

\begin{abstract}
We present data for the dark current of a commercially available CMOS image sensor for different gain settings and bias offsets over the temperature range of 295 to $340 \mathrm{~K}$ and exposure times of 0 to $500 \mathrm{~ms}$. The analysis of hot pixels shows two different sources of dark current. One source results in hot pixels with high but constant count for exposure times smaller than the frame time. Other hot pixels exhibit a linear increase with exposure time. We discuss how these hot pixels can be used to calculate the dark current for all pixels. Finally, we show that for low bias settings with universally zero counts for the dark frame one still needs to correct for dark current. The correction of thermal noise can therefore result in dark frames with negative pixel values. We show how one can calculate dark frames with negative pixel count.
\end{abstract}

Keywords: digital images, image correction, dark current, CMOS

\section{INTRODUCTION}

Dark current is a major source of noise in digital imagers. To decrease the generation of dark current, many camera systems are cooled. In some cases, e.g. consumer cameras, a cooling system is not feasible and dark current can become a problem even for short exposures. A standard method for dark current correction is to take a so-called dark frame, an exposure with a closed shutter, either right before or right after the light exposure. This dark frame is subsequently subtracted from the actual image. Previously, ${ }^{1}$ we reported on a method to compute the dark frame for a CCD imager from the actual image which we want to correct for the dark current. This has the following advantages: This correction can be carried out without knowledge of the temperature and without loss of imager time to collect a dark frame. It will lead to improved image quality and will benefit image fidelity. Moreover, it can be done ex post facto and it provides the ability to remove sensor specific information. ${ }^{2}$ It has been surmised that such computations should be feasible for CMOS imagers as well. With the rise of CMOS sensors' importance in consumer electronics, automotive applications, ${ }^{3}$ and security ${ }^{2}$ and with the improvement of CMOS quality, this technique could be broadly applicable. The goal of the present study is to verify the applicability of the image correction algorithm, as previously applied to scientific CCD imagers, to a commercially available CMOS sensor.

The description of the dark current in a CCD is similar to the analysis of the dark current in a diode and requires taking into account several sources of dark current. ${ }^{4,5}$ We showed that a model of two exponential functions describes the dark current for the back-illuminated chip accurately. ${ }^{6}$ The knowledge of how each individual pixel's dark current changes with temperature can be used to calculate artificial dark frames. However, for many cameras the exact chip temperature is not precisely known. Our proposed dark current correction method requires no knowledge of the real chip temperature. For a given exposure time, the dark current of every pixel is characteristic of a specific temperature. The dark current of any pixel can therefore be used as an indicator of the temperature. Impurities in the silicon cause some pixels to have an unusual dark count. ${ }^{7,8}$ These hot pixels have the highest signal-to-noise ratio and are the best temperature sensors. The basic idea is to use the dark current of hot pixels to obtain an indicator of the chip temperature and predict the dark current of all pixels on the chip. Our previous work applied this method to several well-behaved scientific grade CCD imagers. The question remains whether the same or similar method can be applied to CMOS sensors.

Addressing noise in CMOS imagers has a long history, including various hardware designs to minimize noise ${ }^{9,10}$ and modeling based on circuit models. ${ }^{11,12}$ Others have identified different sources of dark current. ${ }^{13}$ The present imaging performance of color CMOS sensors was reported to be inferior compared to high-end CCD sensors due to excessive dark current non-uniformity, conversion gain non-uniformity, image lag, pixel cross-talk, and low quantum efficiency. ${ }^{14}$ Further insights have been obtained by simulations of noise. ${ }^{15,16}$ Techniques for dark current

Sensors, Cameras, and Systems for Industrial/Scientific Applications IX, edited by Morley M. Blouke,

Erik Bodegom, Proc. of SPIE-IS\&T Electronic Imaging, SPIE Vol. 6816, 68160C

(c) 2008 SPIE-IS\&T $\cdot 0277-786 X / 08 / \$ 18$

SPIE-IS\&T/ Vol. 6816 68160C-1 
correction based on the dark count in pixels outside the optically active imager have also been proposed. ${ }^{17}$ In that work, these dark pixels are used as a proxy for the temperature of the chip and the dark current for the whole imager is extrapolated from it. In general, after dark current corrections are applied, all the subsequent image processing can be performed. ${ }^{18}$

First, we will present the protocol for dark current correction. The dark current data and its analysis are presented in the next section. Finally, we describe the results of the image correction on a flat field and show that there is a potential new twist that can extend the range of applicability of the image correction protocol.

\section{DARK CURRENT PROTOCOL}

In order to see what is involved in dark frame computation, we briefly summarize the correction protocol. ${ }^{1}$ A set of dark frames at various temperatures is taken, and the following basic steps are executed. The first step is to locate hot pixels to serve as temperature indicators. These are selected from an image with a sufficiently large dark signal. A hot pixel in this context has a large dark signal compared to its neighboring pixels. The neighboring pixels are of significance because in an actual image containing signal information, they are used to predict the light signal. Removing the light signal works accurately only if there is high degree of correlation between the light signal of the hot pixel and its adjacent pixels. In most images this correlation between adjacent pixels is very high. The hottest pixels are found by ranking the values of:

$\operatorname{hotpix}(x, y)=\sum_{i=-1}^{1} \sum_{j=-1}^{1}$ weight $(i, j) \cdot \operatorname{pixel}(x+i, y+j)$,

where $(x, y)$ are the coordinates of the pixel and $\operatorname{pixel}(x, y)$ is its dark count. Only the immediate neighbors of the hot pixel are considered. However, it is possible to include a wider area and increase the range of the summation. The weight $(i, j)$ depends on the relative location of an adjacent pixel. Various weighting factors can be chosen. The sum of all the weighting factors equals to zero, such that if all nine pixels are equally hot, $\operatorname{hotpix}(x, y)$ is equal to zero. The next step is to calculate the average of $\operatorname{hotpix}(x, y)$ for the $n$ hottest pixels for frames at different temperatures and this average is used as an indicator for the chip's temperature, $T_{\text {Ind }}$. Then, the value of hotpix $(x, y)$ is fitted to, for instance, a quadratic function of the temperature indicator, $T_{\text {Ind }}$. Hot pixels which have a poor goodness of fit are excluded. Next one stores the coordinates of all hot pixels as well as the three fitting parameters in a file which contains all the information to evaluate the temperature of the chip. Note that one does not use the actual temperature (nor does one need to know the actual temperature). The temperature calibration is a function of the dark current in selected temperature-indicator hot pixels.

The next step in the protocol is to determine the counts of all pixels with respect to $T_{\text {Ind }}$. To accomplish this, the counts of each pixel for frames taken at different temperatures, are fitted with a quadratic least squares fit versus the temperature indicator. Since this fit is used later to calculate the dark count, independent from the neighboring pixels, the actual count of the pixels (not the signal with respect to the neighboring pixels) is used to determine the fitting parameters. The three fitting parameters from the quadratic fit can then be saved as images with the same dimensions as the chip. Once the imager is characterized and calibrated with these fitting parameters, dark frames can be calculated almost instantaneously over the whole calibrated temperature range. One can scale dark frames to other exposure times if one assumes the time dependence is linear for the CCD over the used time range.

\section{DARK CURRENT MEASUREMENTS IN THE FIREFLY}

We used a Firefly MV camera with a 1/3" CMOS color sensor manufactured by Point Grey Research, Inc. to analyze the feasibility and the performance of such a dark current correcting algorithm for CMOS sensors. The chip is a double-buffered global-shutter photodiode device $(640 \times 480$ pixels, 6.0 x $6.0 \mu \mathrm{m}$ pixels, manufactured by Micron - MT9V022177ATC). The Firefly camera is well behaved in that after the temperature was stabilized for a sufficient time span, the measurements were repeatable. Automatic exposure control and color conversion were turned off. The camera was placed inside a temperature-controlled chamber.

The Firefly camera allows adjusting various parameters that are often not changed in a scientific CCD. The camera is capable of both 8 and 10 bit linear data acquisition. For this exploratory work, we choose to present exclusively 8 bit data. The 10 bit data behaved similarly to the present data. The gain could be set from 0 to $12 \mathrm{~dB}$ (or $2.8 \mathrm{DN} / \mathrm{e}$ 
to $11.4 \mathrm{DN} / \mathrm{e}$ ) and was adjusted to optimally collect the data. The brightness setting, essentially a bias offset, can be adjusted between 1 and 255. Level "1" resulted in a negative offset for most pixels, only for levels larger than 125 was the offset positive. This has some interesting implications for dark frame correction and will be discussed in detail later. The camera could be set to frame rates of 7.5, 15,30, and $60 \mathrm{~Hz}$. The frame times used in this study, given as the inverse frame rate were therefore given as $16.7 \mathrm{~ms}, 33.3 \mathrm{~ms}, 66.7 \mathrm{~ms}$, and $133.3 \mathrm{~ms}$. Exposure times could be set for each frame rate up to four times the nominal frame time. Both exposure time and the frame time had an impact on the dark count.
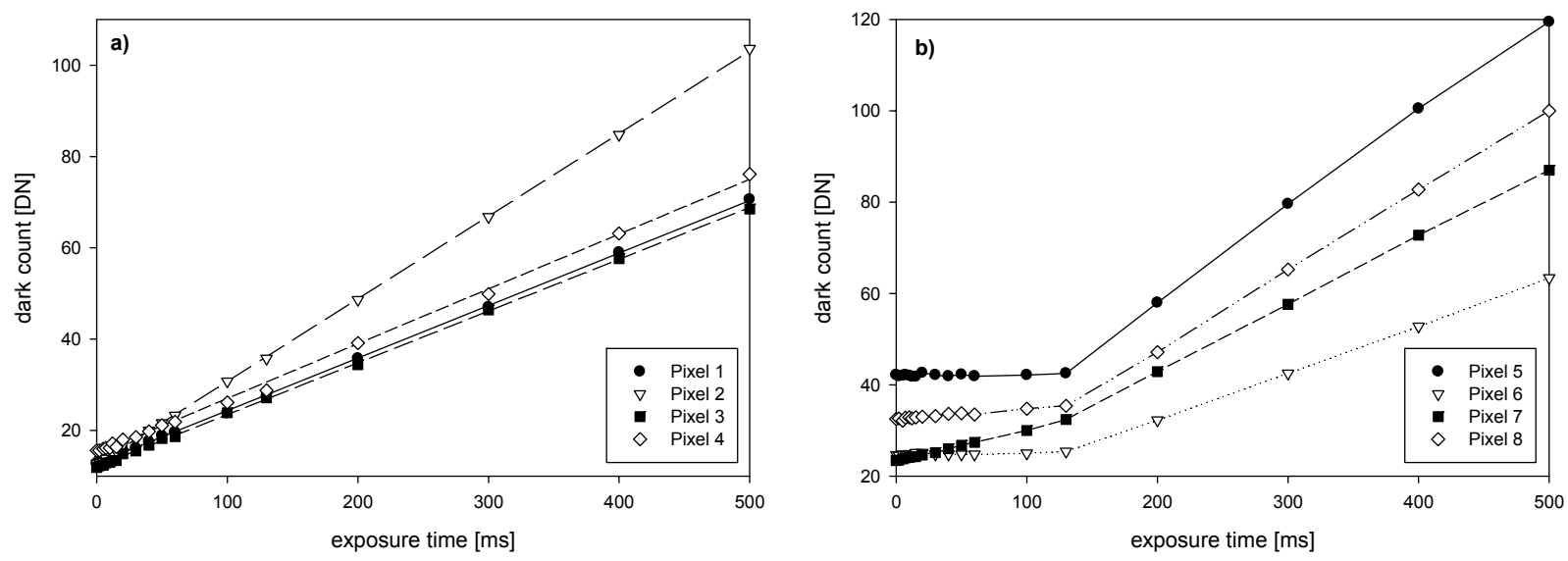

Fig. 1. Dark current vs. exposure time for individual pixels at $35^{\circ} \mathrm{C}, 0 \mathrm{~dB}$, and a frame rate of $7.5 \mathrm{~Hz}$.

(a) with high dark current before the frame time (133.3ms) and (b) data with high dark current after 133.3ms

All data was acquired by taking 10 frames and calculating the average while ignoring the two extremal data points. First we investigated the dependence of the dark current on the exposure time, for times smaller and larger than the nominal frame time. The two panels in Fig. 1 are taken at $35^{\circ} \mathrm{C}$ and a nominal frame rate of $7.5 \mathrm{~Hz}$ corresponding to a frame time of $133.3 \mathrm{~ms}$. Figure 1a shows a set of individual pixels, chosen to be representative of high dark current pixels for short exposure times less than $130 \mathrm{~ms}$. One finds that the dark count increases approximately linearly over the whole range of exposure times. From the slope one gets for example for pixel 2 in Fig. 1a a dark current of about $0.18 \mathrm{DN} / \mathrm{ms}$, approximately 8 times the average dark current at this temperature. Figure $1 \mathrm{~b}$ presents pixels that have a large dark current at exposure times larger than the nominal frame time, but a smaller dark current for times less than $133 \mathrm{~ms}$. The behavior of these pixels is distinctively different. A distinct kink at the frame time can be observed. The dark count for three pixels in Fig. $1 \mathrm{~b}$ is almost constant up to the frame time. Pixel 7 shows a slight increase in dark count $(0.067 \mathrm{DN} / \mathrm{ms})$ for exposure time smaller than the frame time. For exposure times larger than the frame time, the dark current of all four pixels is seen to increase significantly. The slope of the dark count versus exposure time results in a dark current from $0.208 \mathrm{DN} / \mathrm{ms}$ for pixel 5 to $0.102 \mathrm{DN} / \mathrm{ms}$ for pixel 6 . To explain this change, we will assume two distinct regions where dark current is generated. The dark current in the first region is visible for times shorter than the nominal frame time. This is presumed to be the dark current generated in the photodiode. For times larger than the nominal frame time, a second region becomes noticeable. Dark current in this region is generated for all exposure times. However, only for exposure times larger than the frame time does the measured dark signal increases with the exposure time. Unable to find out the exact configuration of the sensor from the manufacturer, we surmised that this location might be the floating diffusion or sense node used in the implementation of the global shutter mode. ${ }^{9}$ If the exposure time is larger than the frame time, the readout is delayed past the $133.3 \mathrm{~ms}$ and more dark current accumulates in the floating diffusion. Up to the frame time, the floating diffusion contributes a constant amount to the total measured dark count. Other pixels that show a substantial dark current at times shorter than $133 \mathrm{~ms}$, show only a small effect when the exposure time is increased beyond $133 \mathrm{~ms}$, or in other words the dark current in the floating diffusion node is small compared to that of the photodiode. However, a careful analysis reveals that almost all of the pixels in this chip have a slight kink at the nominal frame time revealing that most have at least some dark current generated in the sense node. Hence, it is 
necessary to differentiate between dark count generation in the photodiode and dark count generation in the sense node. In this CMOS sensor, one needs to keep track of two dark current generation rates.

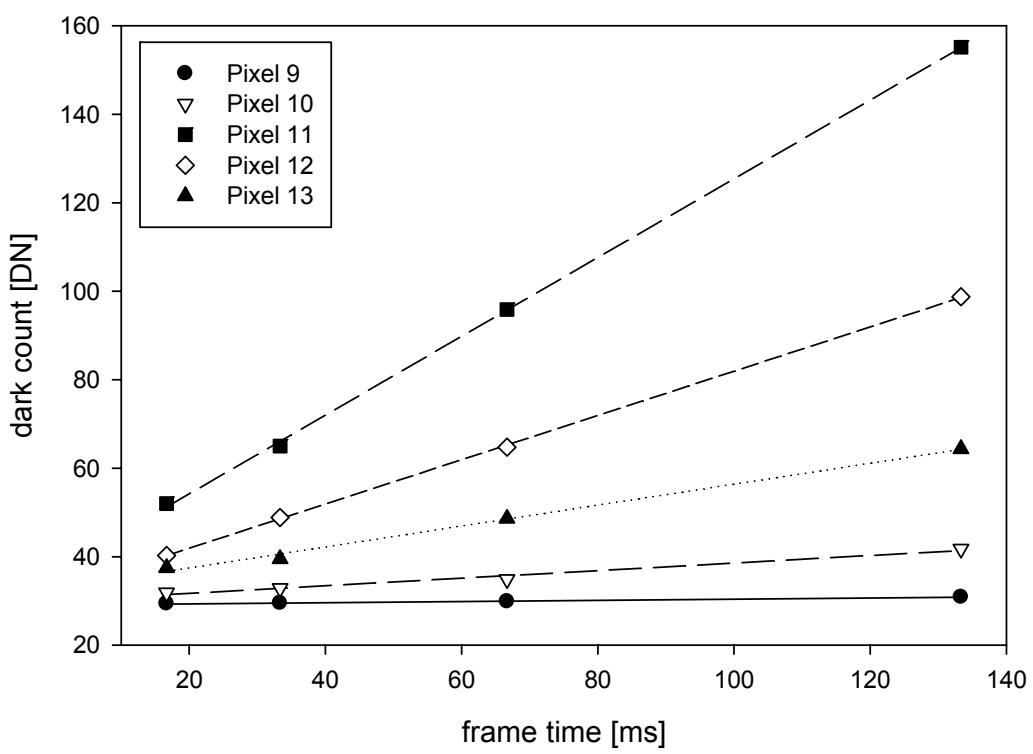

Fig. 2. Dark current vs. frame time (1/frame rate) for selected pixels at $35^{\circ} \mathrm{C}, 12 \mathrm{~dB}$ with an exposure time of $10 \mathrm{~ms}$.

To investigate the dark current generated in two different regions, we varied the frame rate. In Fig. 2, the data for a $10 \mathrm{~ms}$ exposure at the four different frame rates is plotted. Pixels were selected to give a representative sample of dark currents in the sense node. For example, pixel 11 in Fig. 2 produces for the same exposure time of $10 \mathrm{~ms}$ a dark count of $52 \mathrm{DN}$ at $60 \mathrm{~Hz}$ and about 3 times as much $(155.1 \mathrm{DN})$ at $7.5 \mathrm{~Hz}$. The dark count of pixel 9 is almost the same for all four frame rates. One would assume that the dark count collected in the photodiode of a particular pixel is the same for the different frame rates since the exposure time is fixed. The dark current accumulated in the sense node depends linearly on the frame time. As is borne out by the experiments, we see in Fig. 2 that the dark count indeed increases linearly with the frame time.
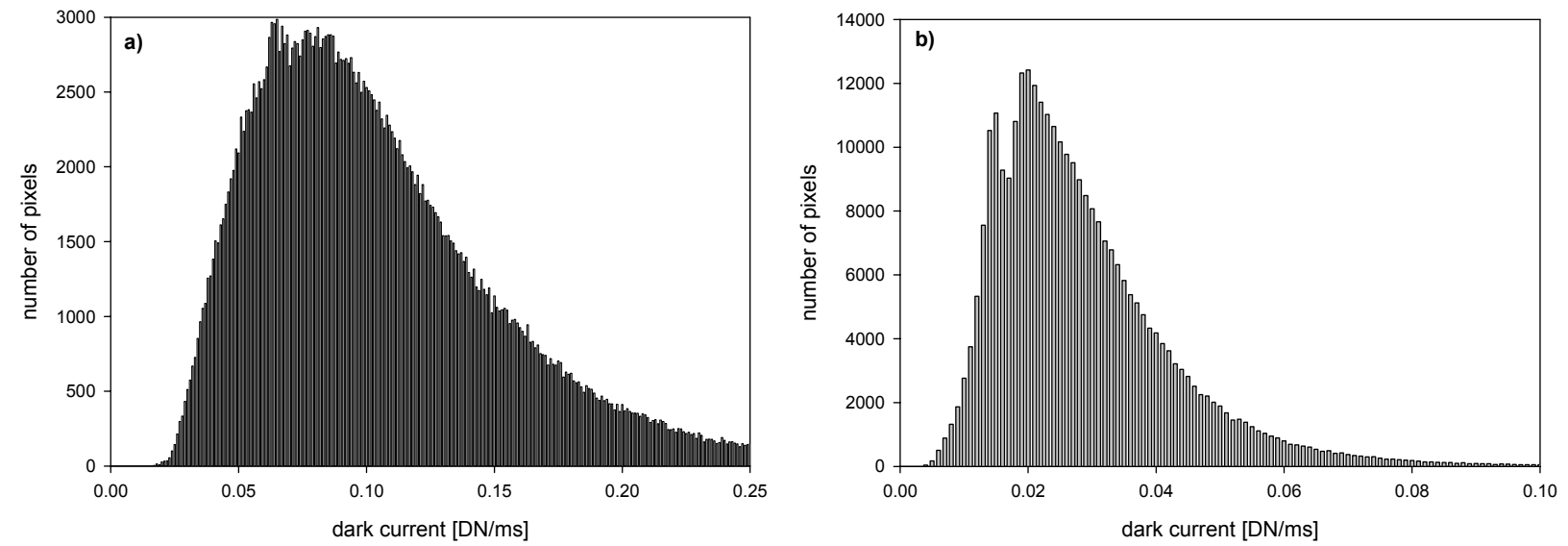

Fig. 3. Histograms of dark current based on exposure times longer than the frame time at $35^{\circ} \mathrm{C}$ : a) with a gain of $12 \mathrm{~dB}$ ) and b) with a gain of $0 \mathrm{~dB}$ (both histograms binned in steps of $0.001 \mathrm{DN} / \mathrm{ms}$ ).

Figure 3a gives the distribution of the dark current, at the largest gain (12 dB), based on a linear fit of the dark count versus exposure time for times longer than the longest frame time $(133.3 \mathrm{~ms})$. The first observation is that there is a 
bimodal distribution. A clearer view of this bimodality shows up at smaller gains. In Fig. 3b, we present the data obtained for a gain of $0 \mathrm{~dB}$. The lesser gain serves to accentuate the two peaks. Secondly, it appears that there is indeed a need for dark current correction. As a first estimate of the impact of this dark current one can find the Gaussian curve fitted to the complete distribution. The distribution shows a peak at $0.08 \mathrm{DN} / \mathrm{ms}$ with standard deviation of about $0.04 \mathrm{DN} / \mathrm{ms}$. Quite a few pixels will fill to $10 \%$ of full well in $100 \mathrm{~ms}$ (about $15 \%$ of the pixels) and another $30 \%$ will fill to $5 \%$ of full well in $100 \mathrm{~ms}$. It has been argued that in a typical camera, fixed pattern noise (FPN) should be less than $0.5 \%$ of full scale. ${ }^{10}$ From the distribution curve, we find that at $100 \mathrm{~ms}$ the FPN will not be acceptable for a consumer product. At higher temperatures, this will be aggravated even more.
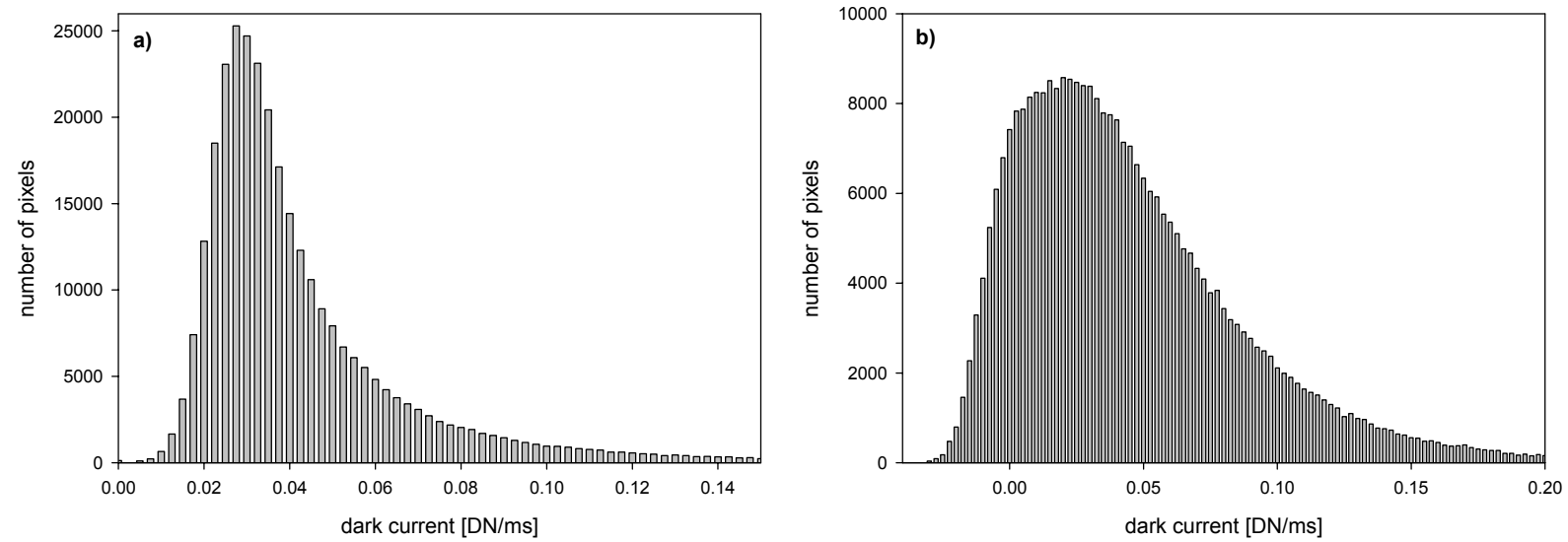

Fig. 4. a) Histogram of dark count slopes $\left(35^{\circ} \mathrm{C}, 12 \mathrm{~dB}, 7.5 \mathrm{~Hz}\right)$ for exposure time data less than the frame time. b) Histogram of dark count slopes vs. the frame times $\left(35^{\circ} \mathrm{C}, 12 \mathrm{~dB}\right.$, exposure time $\left.10 \mathrm{~ms}\right)$. Both histograms binned in steps of $0.0025 \mathrm{DN} / \mathrm{ms}$.

In Fig. 4, we show the dark current distributions for exposure times less than the frame time and for the slopes for the dark counts vs. frame time. A log normal distribution gives a reasonable fit in agreement with the work reported by Baer. ${ }^{15}$ Referring back to Fig. $1 \mathrm{~b}$ and the explanation of the kinks, i.e., the presence of two sources of dark current, one can infer the same from the two histograms. Figure 4a, registers the dark current from the photodiode area and Fig. $4 \mathrm{~b}$ shows the dark current contribution from the sense node. Hence, if one pairs the two independent sources, one would expect them to be additive. To a first approximation the dark count contributions from the photodiode and the sense node are similar, i.e., they have the same mode around $0.03 \mathrm{DN} / \mathrm{ms}$. Since the peak contributions are the same, one would not expect to separate these individual peaks in Figs. 3a and 3b, that is, if only one of the areas contributes, one would see a peak at $0.03 \mathrm{DN} / \mathrm{ms}$. If both contribute, another peak at more than 0.06 $\mathrm{DN} / \mathrm{ms}$ should appear. The net result is that when both currents contribute, another peak should appear in the distribution as is shown in Figs. 3a and 3b.

After having analyzed the dark current of different pixels at different frame rates and exposure times one needs to look at their temperature dependence. Figure 5a shows the dark count of a group of randomly chosen hot pixels in a $30 \mathrm{~ms}$ exposure at various temperatures. The temperatures were measured externally to the sensor and controlled to an accuracy of about $1^{\circ} \mathrm{C}$. The dark count increases as expected and the quadratic curve fits to the pixels show the overall tendency. However, the external temperature might not exactly be the temperature of the sensor. For example, one sees in Fig. 5a that the dark count at $313 \mathrm{~K}$ is higher than expected from the other temperatures. Using hot pixels as a temperature indicator on other hand, avoids the uncertainties in such temperature readings. Fig. $5 \mathrm{~b}$ shows the response of all pixels as a function of the dark count of pixel d. One sees that the dark count of all pixels follow a well defined almost linear relationship. The dark count of pixel $d$ is a much better indicator of the temperature as the externally measured temperature. Hence, a temperature indicator, calculated from the dark current of hot pixels, is a proxy for the real temperature that can be used to predict the dark count of all pixels on the CMOS sensor.

It is further interesting to notice that pixels that behave like pixels 5, 6, or 7 in Fig. $1 \mathrm{~b}$ can be used as temperature indicators for exposure times smaller than the frame time without the need of adjusting for different exposure time 
setting. That is their dark count will not need to be scaled and $T_{\text {ind }}$ for a $30 \mathrm{~Hz}$ exposure will be the same, e.g., for an exposure time of $10 \mathrm{~ms}$ and $30 \mathrm{~ms}$
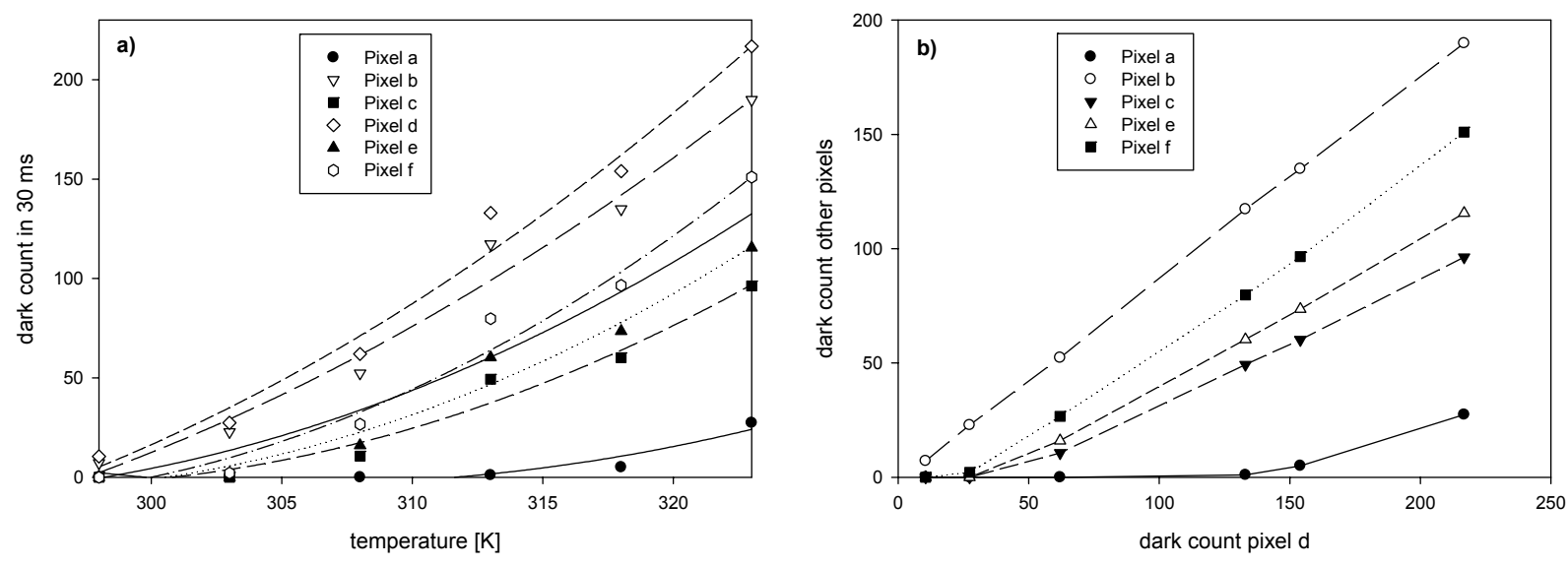

Fig. 5. a) Dark current as function of temperature for several hot pixels (30ms, $12 \mathrm{~dB}, 7.5 \mathrm{~Hz})$. b) dark count of pixel d in Fig. 5 a vs. the other pixels in Fig. 5 a.

\section{IMAGE CORRECTION}

For any camera system the dynamic range is one of the most important parameters. To utilize the maximum dynamic range one can adjust the bias offset depending on the illumination. For an image with a high enough signal it can be beneficial to adjust the bias offset to a negative number. That works without any loss of information as long as the minimum signal is large enough to overcome the bias offset and cause a positive count for all pixels on the chip. For the Point Grey camera, the bias offset can be adjusted from about -32 DN for a brightness setting of 1 to $+37 \mathrm{DN}$ for a brightness setting of 255 . For example, with a brightness setting of 1 and a light intensity that would cause $3.2 \mathrm{DN} / \mathrm{ms}$ the response of a pixel would be zero up to an exposure time of 10 ms. Only for exposures longer than $10 \mathrm{~ms}$ would the pixel actually give a non-zero reading.

The question we would like to address here is what happens to the dark frame when one takes an image with negative bias offsets. For many pixels the dark current will be too small to reach $32 \mathrm{DN}$ for a given exposure time. For example the average dark current at $35^{\circ} \mathrm{C}$ is equal to $0.075 \mathrm{DN} / \mathrm{ms}$ and most pixels will not exceed $32 \mathrm{DN}$ if the exposure time is less than $400 \mathrm{~ms}$. Therefore, a dark frame of, for example, $50 \mathrm{~ms}$ will display zero counts for an average pixel. The actual average dark count generated in $50 \mathrm{~ms}$ is still given by multiplying the dark current with the exposure time: $0.075 \mathrm{DN} / \mathrm{ms}$ times $50 \mathrm{~ms}=3.75 \mathrm{DN}$. For a hot pixel with a dark current of $0.5 \mathrm{DN} / \mathrm{ms}$ the dark count would be $25 \mathrm{DN}$, significantly higher than for an average pixel. However, both pixels would show up with a zero count in the dark frame. Therefore, to accurately correct for dark current, dark frames have to be obtained starting with a value equal to the bias offset. Even if this might cause the somewhat surprising result of a dark frame with a large number of pixels that have a negative dark count. One can obtain such a dark frame by:

1.) Calculating dark current as the counts versus exposure time of an image with a large brightness setting.

2.) Obtaining the correct bias offset from the y-intercept of a linear fit versus exposure time for light exposures with the correct brightness setting (in our case 1).

The dark count for brightness of 1 is then equal to the dark current in DN/ms (from step 1) times the exposure time plus the negative bias offset (from step 2).

Panel a in Fig. 6 shows the histogram of a dark frame calculated as described above at $35 \mathrm{C}, 50 \mathrm{~ms}, 7.5 \mathrm{~Hz}$ and a brightness setting of 1 . The distribution, as is typical for a dark frame, is skewed to right. Unlike a typical dark frame, the average value is negative $(-28.6 \mathrm{DN})$ and most of the other pixels have negative dark count values as well.

To test the dark current correction we obtained flat fields at the same setting as the dark frame. Since the Point Grey camera has a color chip with a Bayer RGB filter pattern the individual color channels have to be analyzed 
individually. Panel $b$ shows pixel values of all green pixels (half of the pixels in the imager are green). One can see that the light intensity was high enough that all pixels reached a positive count. However, one would expect a Poisson like distribution for a flat field exposure. Due to the dark current the distribution is skewed to the right. Subtracting a regular dark frame will not improve the situation, since most pixels will have a zero value. Using the dark frame obtained with the method described above one can do an accurate dark current correction. Panel $\mathrm{c}$ of Fig. 6 shows the histogram for the flat field after subtraction of the accurate dark frame (see Panel a for the values of the dark frame). As one would expect the distribution for the flat field is symmetrical with pixel values close to the average value. The average pixel value is given as $54.9 \mathrm{DN}$, larger than the average value before the dark current correction. The width of the distribution has decreased significantly. The standard deviation of $7.62 \mathrm{DN}$ for the uncorrected frame has decreased to $1.39 \mathrm{DN}$ of the corrected frame.
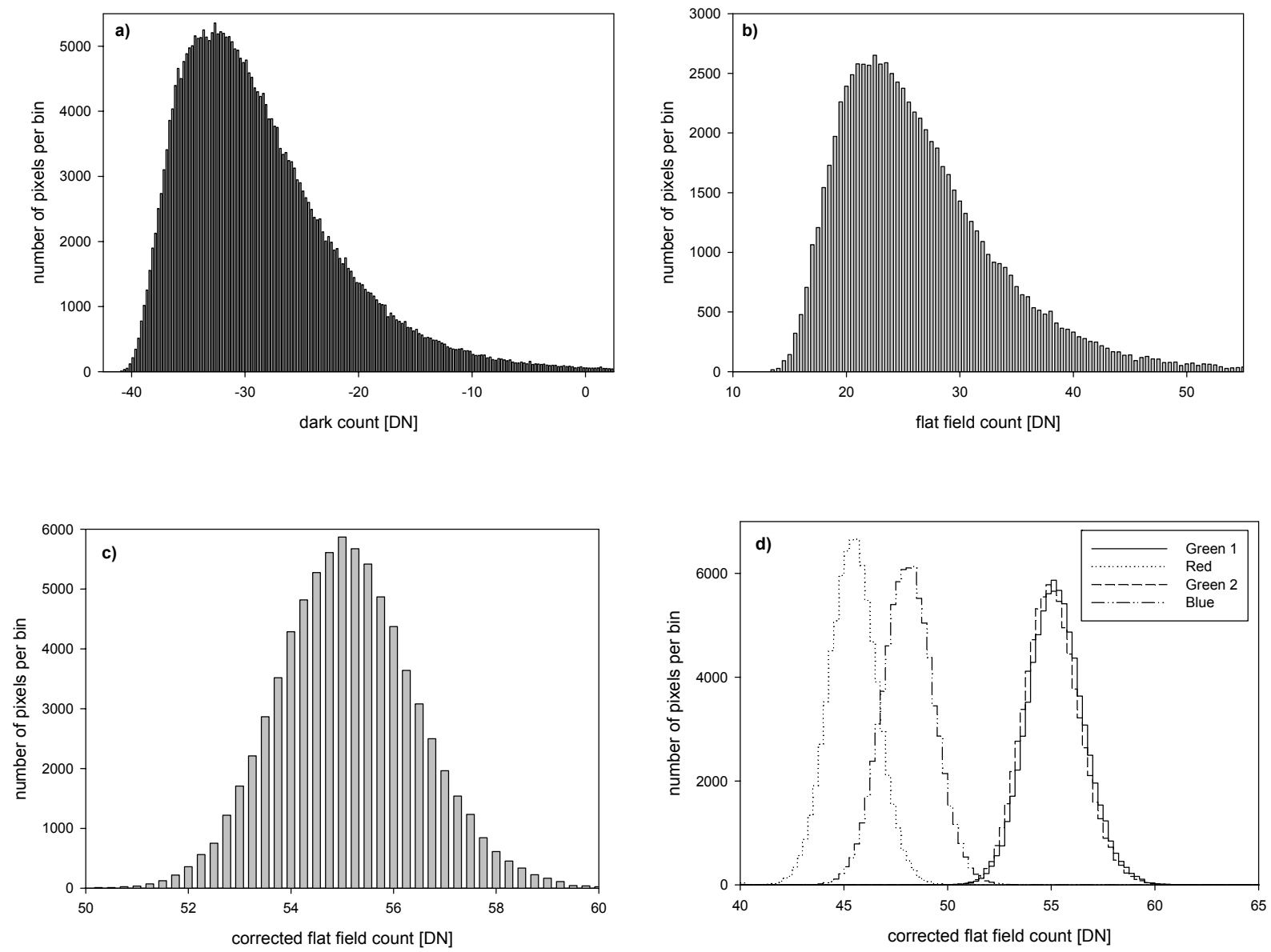

Fig. 6. Dark current correction of flat fields with a negative bias offset $\left(35^{\circ} \mathrm{C}, 7.5 \mathrm{~Hz}, 50 \mathrm{~ms}\right.$, and brightness $\left.=1\right)$. a) calculated dark frame distribution for the complete chip, b) pixel values of half of the green pixels in the flat field, c) the same green pixel flat minus the appropriate dark frame, d) corrected flats of all pixels grouped by the Bayer array.

Of course, the same correction can be applied to the red and blue pixels. Panel $\mathrm{d}$ shows the pixel values for all pixels. The first peak corresponds to pixels with a blue filter, the middle peak to red pixels, and the right peak is for pixels with a green filter. 


\section{SUMMARY}

We have demonstrated that all the ingredients for dark frame correction exist in a CMOS sensor. ${ }^{19}$ These ingredients include the presence of hot pixels that depend strongly on temperature that can serve as a temperature indicator and a predictable behavior of the dark current for those pixels not chosen for their hotness. We have shown that a flat field corrected by a calculated dark frame, albeit without calculating its temperature using hot pixels, produced a corrected flat field that is significantly better. Because there are two kinds of sources of dark current, one which depends solely on exposure time and the other which depends on frame time, it should, in principle, be possible to calculate temperature and exposure time. Since the protocol allows for the correction of images for dark current over a range of temperatures, it should prove useful in situations where the temperature of the sensor is elevated, for instance in automotive applications.

\section{REFERENCES}

${ }^{1}$ R. Widenhorn, A. Rest, M.M. Blouke, R.L. Berry, and E. Bodegom, “Computation of Dark Frames in Digital Imagers,” Proc. of SPIE 6501, 650103 (2007)

${ }^{2}$ J. Lukas, J. Fridrich, and M. Goljan, “Digital camera identification from sensor pattern noise,” IEEE Trans. Information

Forensics and Security, 1, 205 (2006)

${ }^{3}$ B.J. Hosticka et al, "CMOS Imaging for Automotive Applications," IEEE Trans. Electron Devices, 50-1, 173, (2003)

${ }^{4}$ A. S. Grove, "Physics and Technology of Semiconductor Devices," (John Wiley \& Sons, 1967)

${ }^{5}$ S.M. Sze, "Physics of Semiconductor Devices," second edition (John Wiley \& Sons, 1981)

${ }^{6}$ R. Widenhorn, M. M. Blouke, A. Weber, A. Rest, and E. Bodegom, “Temperature dependence of dark current in a CCD,” Proc. SPIE Int. Soc. Opt. Eng. 4669, 193 (2002)

${ }^{7}$ W. C. McColgin, J. P. Lavine, and C. V. Stancampiano, "Probing metal defects in CCD image sensors," Mat. Res. Soc. Symp. Proc. 378, 713 (1995)

${ }^{8}$ W. C. McColgin, J. P. Lavine, C. V. Stancampiano, and J. B. Russell, “Deep-level traps in CCD image sensors,” Mat. Res. Soc. Symp. Proc. 510, 475 (1998)

${ }^{9}$ G. Yang, O. Yadid-Pecht, C. Wrigley, and B. Pain, “A Snap-Shot CMOS Active Pixel Imager for Low-Noise,” High-speed Imaging, IEDM 98, 45 (1998)

${ }^{10}$ M.F. Snoeij, “Analog Signal Processing for CMOS Image Sensors,” PhD Dissertation, TU Delft (2007)

${ }^{11}$ B. Pain, T. Cunningham, B. Hancock, C. Wrigley, and C. Sun, "Excess Noise and Dark Current Mechanism in CMOS Imagers," Proc. Int. Image Sensor Workshop 145, (2007)

${ }^{12}$ H. Tian, B. Fowler, and A. El Gamal, “Analysis of Temporal Noise in CMOS Photodiode Active Pixel Sensor,” IEEE Journal of Solid-State Circuits 36, 92 (2001)

${ }^{13}$ H. In Kwon, I.M. Kang, B.G. Park, J.D. Lee, S.S. Park, "The Analysis of Dark Signals in the CMOS APS imagers from the Characterization of Test Structures," IEEE Trans. Electron Dev. 51, 178 (2004)

${ }^{14}$ A.J. Blanksby and M.J. Loinasz, "Performance Analysis of a Color CMOS Photogate Image Sensor," IEEE Trans. Electron Dev. 47, 55 (2000)

${ }^{15}$ R.L. Baer, “A Model for Dark Current Characterization and Simulation,” Proc. of SPIE 6501, 606805 (2007)

${ }^{16}$ S.H. Lim, "Characterization of Noise in Digital Photographs for Image Processing," Proc. of SPIE 6069, 606900 (2006)

${ }^{17}$ R.L. Baer, "Efficient Dark Current Subtraction in an Image Sensor," US Patent 6,714,241 (2004)

18 A.J.P. Theuwissen, "Image Processing Chain in Digital Still Cameras," VLSI Circuits, Digest of Technical Papers, 2 (2004)

${ }^{19}$ R. Widenhorn, A. Rest, R.L. Berry, and E. Bodegom, patent pending 